\title{
Fiducial Interval Estimation of Scale Parameter of Lognormal Population Distribution
}

\author{
Li Xiuzhen $^{*}$ \\ Media and Mathematical institute \\ Jiliin Engineering Normal University \\ JiLin China \\ e-mail: arnold0110@sina.com \\ * Corresponding Author \\ Ma Yanying \\ Media and Mathematical institute \\ Jiliin Engineering Normal University \\ JiLin China \\ Zhang Na \\ College of Art and Design \\ Shenyang Jianzhu University \\ Shen Yang, China
}

\begin{abstract}
Using a new method to solve fiducial interval estimation of parameter, the method of fiducial inference can obtain the fiducial interval estimation of scale parameter of lognormal population distribution.the method intuitive novelty special.But fiducial distribution maybe different if use different inference method while looking for fiducial distribution, this cause the interval of the parameter that we achieved not unique. We can receive very similar interval estimation by use of fiducial inference and Neyman's method, sometimes even exactly the same, but it is completely different in explanation; while sometimes the result we received by use of fiducial inference is better than use of Neyman's theory, even can solve the problem that the theory of Neyman can not solve.All these fully shown the special and novelty of the method.
\end{abstract}

Keywords-lognormal population; fiducial distribution; interval estimation; method of fiducial inference ;scale parameter

\section{INTRODUCTION}

In the statistics, the parameter rate of population distribution can be divided into point estimation and interval estimation, but the interval estimation of parameter can be divided into asymptotic interval estimation and exact interval estimation again, based on different statistical methods exact interval estimation can be divided into confidence interval、 tolerate interval、 fiducial interval、confidential interval, bayes estimation interval、nonparametric estimation interval etc. Among them fiducial interval estimation is a new method to solve the problem of interval estimation ----- fiducial inference, which is proposed by Fisher in the nineteen thirties through broaden the idea of maximum likelihood estimation. The important thought of this method lies in when not involve the prior distribution of parameters , base on the sample observations $\mathrm{x}$ then give a distribution

\author{
Li Quanquan \\ mathematic room \\ Guo jiadiansenior middle school \\ JiLin China \\ Zhu Lisa \\ Media and Mathematical institute \\ Jiliin Engineering Normal University \\ JiLin China
}

of parameter----- fiducial distribution, fiducial interval estimation is based on fiducial distribution. Mathematical speaking, fiducial distribution has the quality of probability distribution, but it not permit any frequency explains, it only expressed : based on the informations of sample observations, "confidential degree" of parameter falls in each range, so Fisher called this distribution as fiducial distribution. Because of fiducial inference's special thought 、 novel method 、 difficult inference, hardly have the book introduce the contents of it, the related article is also limited.

Lognormal population distribution is important object of study.It has widely application value in practial.For example :analgesia effect of acupuncture anesthesia ,the length of english words, how long time epidemic spread,some electrical lifetime etc,whatsmore using lognormal distribution substituted normal distribution in contemporary finance as assets price distribution established the theorem, which is reasonable and wery beautiful.The importance of lognormal distribution is improving. It discussed combination confidence region of lognormal population in [1],but it not discussed the fiducial interval of lognormal population This paper discussed fiducial distribution and fiducial interval estimation of scale parameter of lognormal population, receivd the conclusion that confidence interval of parameter is similar to fiducial interval of parameter.

\section{RELATED THEOREM AND LEMMA}

In the 1930s, Fisher puted forward the method of fiducial inference by using the though of maximum likelihood estimation. He thought there will be a distribution of parameter $\theta$,afer gain the observed 
value of sampleX.The distribution showed the "believable degree"that $\theta$ fall in each range.Fisher called it fiducial distrbution.

Because fiducial distrbution.is difficult to understand,so I introduce a example here

\section{A. For example}

SupposeX $=\left(X_{1}, X_{2}, \ldots X_{n}\right)$ are simples random sample that from normal population $N(\mu, 1)$, which $-\infty<\mu<\infty$,because $\bar{X}$ is sufficient statistic for $\mu$, so we consider the question base on $\bar{X}$, because $\bar{X} \sim$ $N\left(\mu, \frac{1}{n}\right)$ so if we denoted as $\quad e \sim$ $N(0,1), \quad$ then we get

$$
\bar{X}=\mu+\frac{1}{\sqrt{n}} \cdot e
$$

Transpose upside algebraic expression we can get $\mu=\bar{X}-\frac{1}{\sqrt{n}} \cdot e$

(2)is the equivalent transformation from(1), but Fisherizgive(2) a new explanation different completely:he think after obtained observed value $\mathrm{X}$ of sample, and obtained $\bar{X}$ further,(2)give out the distribution of $\mu$,

$$
\mu \sim N\left(\bar{X}, \frac{1}{n}\right), \text { Fisher called the distribution }
$$
as fiducial distribution of $\mu$,through the fiducial distribution of $\mu$ can get

$$
P\left(\bar{x}-\frac{1}{\sqrt{n}} \cdot u_{1-\frac{\alpha}{2}} \leq \mu \leq \bar{x}+\frac{1}{\sqrt{n}} \cdot u_{1-\frac{\alpha}{2}}\right)=1-\alpha
$$

Which $\mathbf{1}-\alpha$ is called fiducial level.

$$
\left[\bar{x}-\frac{1}{\sqrt{n}} \cdot u_{1-\frac{\alpha}{2}}, \bar{x}+\frac{1}{\sqrt{n}} \cdot u_{1-\frac{\alpha}{2}}\right] \text { is called fiducial }
$$

interval of $\mu$ that fiducial level is $\mathbf{1}-\alpha$.

There are several methods about inducement of fiducial distribution, but established function model to induce fiducial distribution is a simple way.

\section{B. Definition of function model}

there are three elements for function model, observed value $\mathrm{X}$, take value from $\left(\mathrm{R}, \beta_{x}\right)$; parameter $\theta$, take value from $\left(\Theta, \beta_{\Theta}\right)$; error variance e, take value from $\left(\varepsilon, \beta_{\varepsilon}\right)$; distribution of e is $\mathrm{P}, \mathrm{P}$ have nothing to do with $\theta$.Among three quantities the functional relationship is existed: $(\theta, \mathrm{e}) \rightarrow \mathrm{X}$,

denoted as $\mathrm{X}=\theta \circ \mathrm{e}$, which "०" expressed some operation .Solving each $\mathrm{X} \in \mathrm{R}$, existence $\theta$ and

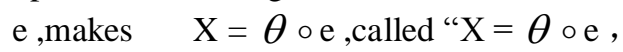

$\mathrm{e} \sim \mathrm{P}$ "function model.In function model when $\theta$ is given , $\mathrm{X}$ is the function for e,then through the model can pushe out the distribution of $\mathrm{X}^{\prime \prime} \mathrm{X} \sim P_{\theta}(x)$ ". So when $\theta$ is given,through the function model "X $=\theta$ ○ e , e $\sim \mathrm{P}$ ",we can induce a statistical structure: $\mathrm{X} \sim P_{\theta}(x), \quad \theta \in \Theta$. Besides, if for all the $\mathrm{X}$ and $\mathrm{e}$, a unique $\theta$ is existed, makes $\mathrm{X}=\theta \circ$ e.Then $\theta$ is the function for $\mathrm{e}$, after gain the observed value of sample $\mathrm{X}$.Thus through function model" $\mathrm{X}=\theta \circ \mathrm{e}, \mathrm{e} \sim \mathrm{P}$ " induce distribution of $\theta$,this is fiducial distribution of $\theta$.For given $\mathbf{0}<\alpha<\mathbf{1}$,fiducial interval $\left[\hat{\sigma}_{L}^{2}, \hat{\sigma_{U}^{2}}\right]$ is exsitent, make $\mathrm{p}\left\{\hat{\sigma}_{L}{ }^{2}<\sigma^{2}<\hat{\sigma}_{U}{ }^{2}\right\}=1-\alpha$,call the $\left[\hat{\sigma}_{L}^{2}, \hat{\sigma_{U}^{2}}\right]$ fiducial interval of fiducial level is $\mathbf{1}-\alpha$.

\section{Definition of lognormal population distribution 1:Suppose population X obey lognormal distribution, it's density function of distribution is}

$$
f\left(x ; \mu, \sigma^{2}\right)= \begin{cases}\frac{1}{\sqrt{2 \pi} \sigma x} e^{-\frac{(\ln x-\mu)^{2}}{2 \sigma^{2}}} & x>0 \\ 0 & x \leq 0\end{cases}
$$

Which $-\infty<\mu<\infty, \sigma^{2}>0$

lognormal distribution is called lognormal distribution because the variable $\ln x$ obey normal distribution $N\left(\mu, \sigma^{2}\right)$ so we call it lognormal distribution.

In this artical i only study single parameter's fiducial interval estimation, so Suppose the other parameter is known,the definition is as follow

\section{Definition of lognormal population 2:}

If population $\mathrm{X}$ meet $\ln \mathrm{x} \sim \mathrm{N}\left(\mu, \sigma^{2}\right)$,

that is $\quad \mathrm{X} \sim \mathrm{f}(\mathrm{x})=\frac{1}{\sqrt{2 \pi} \sigma} e^{-\frac{(\ln x-\mu)^{2}}{2 \sigma^{2}}}$

which $\mathrm{x}>0,0<\sigma^{2}<\infty, \mu$ is known .

Then called $\mathrm{X}$ lognormal population. 
Suppose $X_{1}, X_{2}, \ldots X_{n}$ are simple random sample that from lognormal population $X$, denoted as $Q^{2}=\sum_{i=1}^{n}\left(\ln X_{i}-\mu\right)^{2}$

\section{E. Related lemmas}

Suppose $X_{1}, X_{2}, \ldots X_{n}$ are simples random sample that from lognormal population $X$,

Then

(i) $Q^{2}$ is sufficient statistic for $\sigma^{2}$ (ii) $\frac{Q^{2}}{\sigma^{2}} \sim \chi^{2}(n)$

\section{MAIN RESULTS}

Theorem 1 : Suppose $X$ is lognormal population. $X_{1}, X_{2}, \ldots X_{n}$ are simple random samples, $\sigma^{2}$ is unknown scale parameter, $\mu$ is known,for $\mathbf{0}<\alpha<\mathbf{1}$, then interval estimation of fiducial level $\mathbf{1}$ $-\alpha$ for $\sigma^{2}$ is $\quad\left(\frac{S^{2}}{\chi_{1-\frac{\alpha}{2}}^{2}(n)}, \quad \frac{S^{2}}{\chi_{\frac{\alpha}{2}}^{2}(n)}\right)$

Proving: because $Q^{2}=\sum_{i=1}^{n}\left(\ln X_{i}-\mu\right)^{2}$ is sufficient statistic for $\sigma^{2}$,so based on the principle of full disclose, we considering fiducial interval estimation of $\sigma^{2}$ by $Q^{2}$, because $\frac{Q^{2}}{\sigma^{2}} \sim \chi^{2}(n)$, so denoted as $\mathrm{e} \sim \chi^{2}(n), \quad$ then gain the function model

$$
Q^{2}=\sigma^{2} \text {. e }
$$

finishing it ,then get $\sigma^{2}=\frac{Q^{2}}{e}$, that is after we gain the observed value $\mathrm{X}$ of sample, then we get $Q^{2}$,furthermore get the fiducial distribution of $\sigma^{2}$,

$$
\frac{\sigma^{2}}{Q^{2}} \sim \frac{1}{e}, \mathrm{e} \sim \chi^{2}(n)
$$

So the fiducial distribution of $\sigma^{2}$ is inverted $\chi^{2}$ distribution that degree of freedom is $\mathrm{n}$. Thus gain interval estimation of fiducial level $1-\alpha$ for $\sigma^{2}$ is $\left[\hat{\sigma}_{L}^{2}, \hat{\sigma_{U}^{2}}\right]$
Base on the demand that the interval length should be shortest ,the left endpoint $\hat{\sigma_{L}^{2}}$ and the right endpoint $\hat{\sigma_{U}^{2}}$ should meet certain condition,but because

$\chi^{2}$-distribution is single peaka symmetric distribution,so it is hard to lookup to gain,so we can consider equal tail fiducial distribution then $\chi_{\frac{\alpha}{2}}^{2}(n) \leq e \leq \chi_{1-\frac{\alpha}{2}}^{2}(n)$

By the fiducial distribution of $\sigma^{2}$ we can gain $\mathrm{p}\left\{\chi_{1-\alpha / 2}^{2}(\mathrm{n})<\frac{\sum_{\mathrm{i}=1}^{\mathrm{n}}\left(\ln X_{\mathrm{i}}-\mu\right)}{\sigma^{2}}<\chi_{\alpha / 2}^{2}(\mathrm{n})\right\}=1-\alpha$

( $1-\alpha$ is called fiducial coefficient )

That is we can gain interval estimation of fiducial level $1-\alpha$ for $\sigma^{2}$

$$
\left(\frac{S^{2}}{\chi_{1-\frac{\alpha}{2}}^{2}(n)}, \quad \frac{S^{2}}{\chi_{\frac{\alpha}{2}}^{2}(n)}\right)
$$

(Annotation:this is not the optimal interval estimation)

\section{REFERENCES}

[1] Mao Shi-song,Wang Jing-long,Pu Xiao-long. Advanced Mathematical Statistics [M]. higher education publishing house , 1998

[2] Chen Xi-ru.,Mathematical Statistics Introduction [M]. science press, 1981.

[3] Chen Guang-shu.Combination confidence region of lognormal population distribution [ J]

[4] Fei He-liang, Xu Xiao-ling.Combination confidence region of three parameters of weibull distribution[J]. Application probability statistics, 1992 (4)

[5] Gao Shang.Calculation of shortest confidence interval[J].Journal of east china shipbuilding institute, 2003.

[6] Dawid A P and Stone M.The functional-model basis of fiducial inference (with discussion).Ann Statist,1982,10:01054-1074

[7] Dawid AP and Wang J L. Fiducial prediction and semi-bayesion infetence. Ann Statist, 1993,21:1119-1138.

[8] Gunnar B. Probability and statistics Theory and applicationas. Newyork springer, 1989.

[9] Chen Xi-ru.Probability Theory and Mathematical Statistics [M].University of Science and Technology of China publishing house, 1992.

[10] Li Xin-min,Li Guo-ying.Confidence interval of variance ratio inmixed linear model [J].Application probability statistics, 2004.

[11] Liu Xu-hua,Xu Xing-zhong,Li Na.Generalized confidence intervalof a kind of availability of repairable system steady .[J] Journal of Bei jing university of technology 2010,(05) 